\title{
Risk-adjusted antibiotic consumption in 34 public acute hospitals in Ireland, 2006 to 2014
}

A Oza ${ }^{1}$, F Donohue ${ }^{2}$, H Johnson ${ }^{2}$, R Cunney ${ }^{1}$

1. Health Service Executive (HSE) Health Protection Surveillance Centre (HPSC), Dublin, Ireland

2. Knowledge Management (incorporating Health Intelligence), Health Service Executive (HSE) Health and Wellbeing Directorate, Dublin, Ireland

Correspondence: Ajay Oza (ajay.oza@hse.ie)

As antibiotic consumption rates between hospitals can vary depending on the characteristics of the patients treated, risk-adjustment that compensates for the patient-based variation is required to assess the impact of any stewardship measures. The aim of this study was to investigate the usefulness of patient-based administrative data variables for adjusting aggregate hospital antibiotic consumption rates. Data on total inpatient antibiotics and six broad subclasses were sourced from 34 acute hospitals from 2006 to 2014 . Aggregate annual patient administration data were divided into explanatory variables, including major diagnostic categories, for each hospital. Multivariable regression models were used to identify factors affecting antibiotic consumption. Coefficient of variation of the root mean squared errors (CV-RMSE) for the total antibiotic usage model was very good (11\%), however, the value for two of the models was poor (>30\%). The overall inpatient antibiotic consumption increased from 82.5 defined daily doses (DDD)/100 bed-days used in 2006 to 89.2 DDD/100 bed-days used in 2014; the increase was not significant after risk-adjustment. During the same period, consumption of carbapenems increased significantly, while usage of fluoroquinolones decreased. In conclusion, patient-based administrative data variables are useful for adjusting hospital antibiotic consumption rates, although additional variables should also be employed.

\section{Introduction}

Antibiotic consumption can vary between hospitals depending on a number of factors including implementation and adherence to antibiotic policies, antibiotic resistance rates, and hospital function which depends on the patient characteristics [1-3].

A few reports have focused on risk adjustment models that account for differences in specific health risks that patients bring to their healthcare facilities, thus 'levelling the playing field' when comparing rates of antibiotic consumption between hospitals with varied case mix [4-6]. These approaches provide a benchmarking tool to identify facilities that have consistently higher or lower than expected rates in order to encourage compliance with guidelines. As well as adopting these approaches to the Irish antibiotic consumption context, this study explored how changes in case mix over time can affect antibiotic usage.

Variables relating to a variety of patient characteristics from public acute hospitals, based on administrative data, are readily available in Ireland [7]. Unlike parameters for clinical services (such as provision of intensive care, oncology and cardiac services) which provide a static representation, patient-based parameters (age, sex, place of admission and discharge, diagnoses and procedures) can reflect changes in case mix over time. The rates of antibiotic use in hospitals are dynamic and have been shown to change over time not only in Ireland but in many countries [8]. Hospital administrative data are therefore a good candidate for developing risk adjustment models. The aim of this study was to investigate the usefulness of patient-based administrative data variables for adjusting hospital antibiotic consumption rates.

\section{Methods}

Study design

The study was an observational, retrospective analysis of aggregate data on antibiotic use and patient administration from 34 public acute hospitals in Ireland. The participating hospitals in this study represented all tertiary/referral hospitals in Ireland and all general hospitals bar two facilities that were unable to provide consistent antibiotic consumption data. Single-speciality hospitals (maternity, paediatric or orthopaedic) were excluded. 


\section{FIGURE 1}

Heat map showing percentage of 34 administration variables for antibiotic consumption in public acute hospitals, Ireland, 2006-2014

\section{A. By explanatory variable}

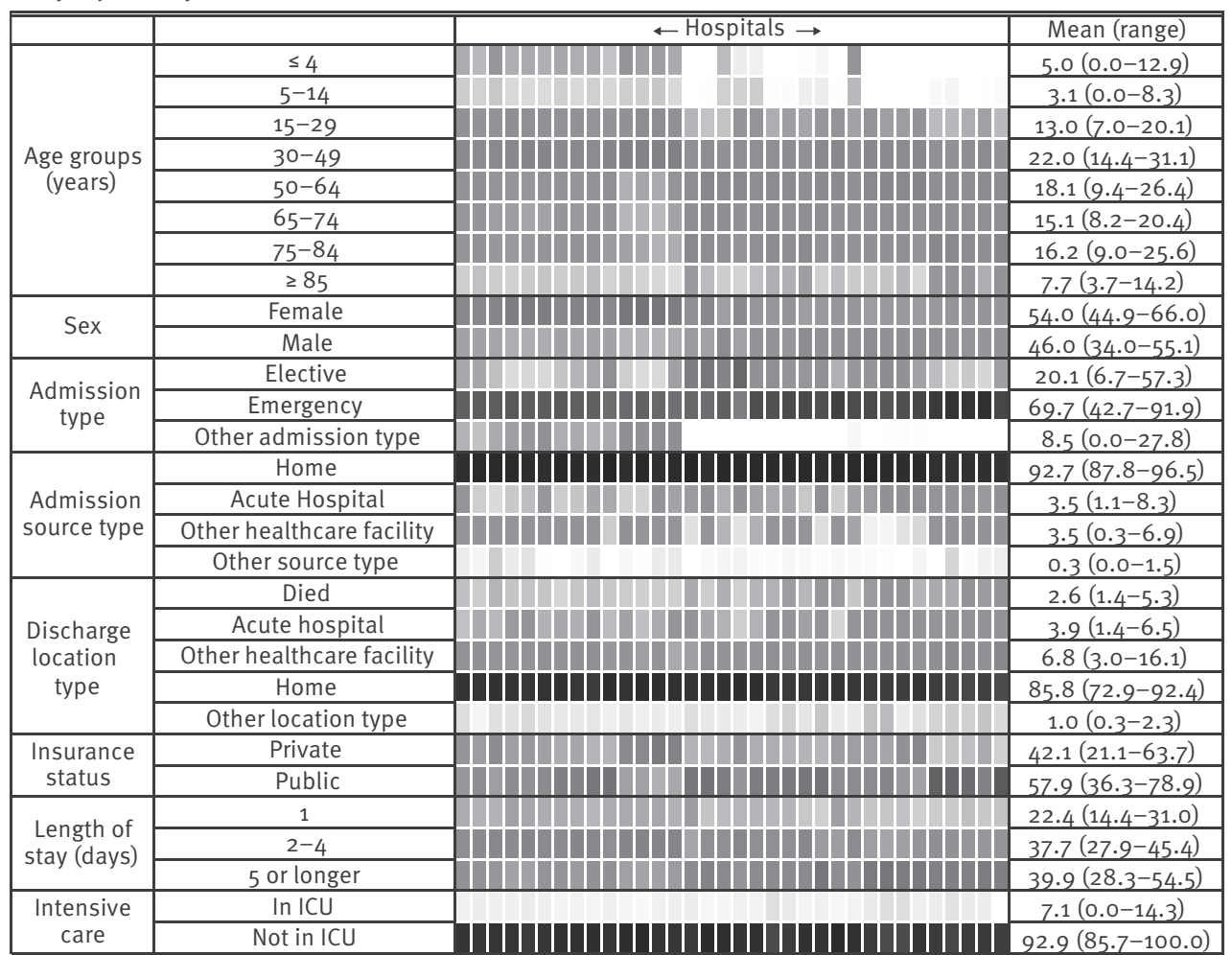

B. By major diagnostic category

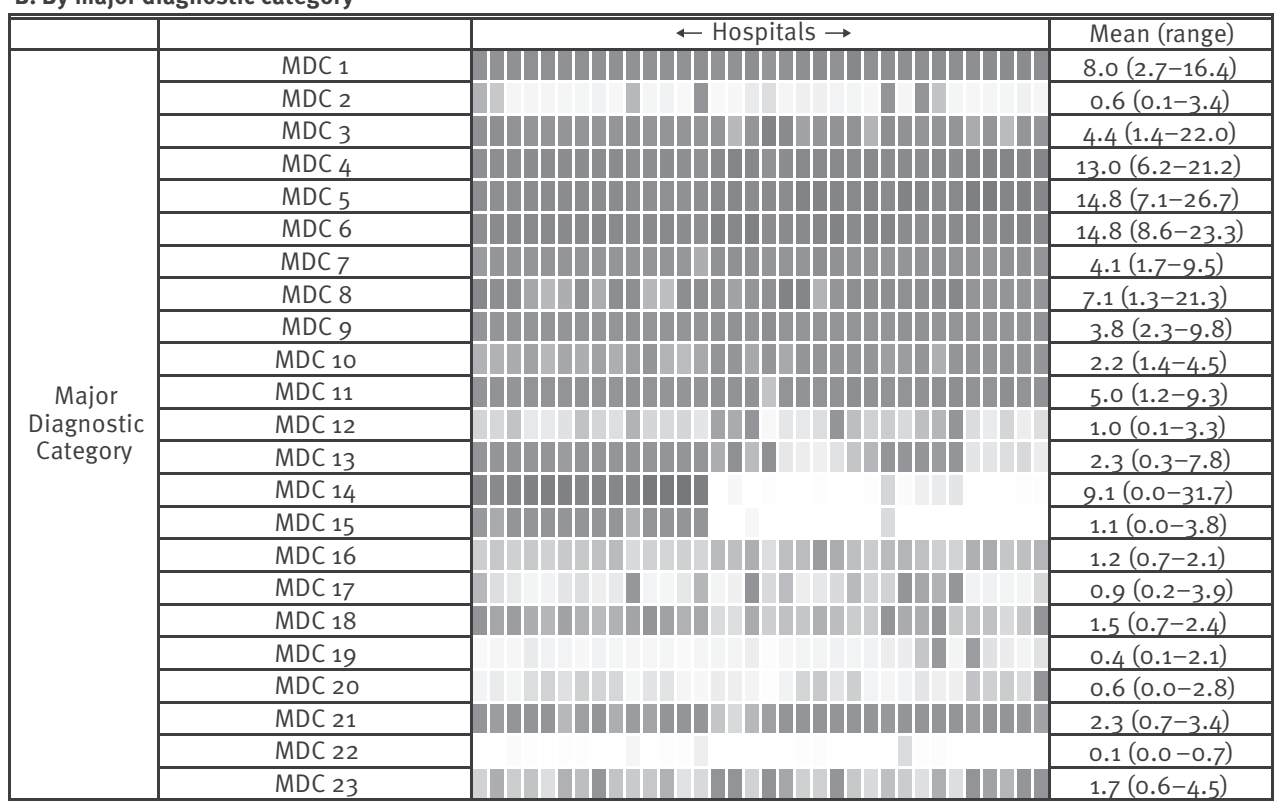

Key

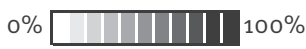

ICU: intensive care unit; MDC: major diagnostic categories.

The variables are averages over the study period. For panel A, they are grouped into eight sections which each total $100 \%$ for any hospital.

MDC 1: diseases and disorders of the nervous system; MDC 2: diseases and disorders of the eye; MDC 3 : diseases and disorders of the ear, nose, mouth and throat; MDC 4: diseases and disorders of the respiratory system; MDC 5 : diseases and disorders of the circulatory system; MDC 6: diseases and disorders of the digestive system; MDC 7: diseases and disorders of the hepatobiliary system and pancreas; MDC 8: diseases and disorders of the musculoskeletal system and connective tissue; MDC 9: diseases and disorders of the skin, subcutaneous tissue and breast; MDC 10: endocrine, nutritional and metabolic diseases and disorders; MDC 11: diseases and disorders of the kidney and urinary tract; MDC 12: diseases and disorders of the male reproductive system; MDC 13: diseases and disorders of the female reproductive system; MDC 14: pregnancy, childbirth and the puerperium; MDC 15: newborns and other neonates; MDC 16: diseases and disorders of the blood and blood forming organs and immunological disorders; MDC 17: neoplastic disorders (haematological and solid neoplasms); MDC 18: infectious and parasitic diseases; MDC 19: mental diseases and disorders; MDC 20: alcohol/drug use and alcohol/drug-induced organic mental disorders; MDC 21: injuries, poisoning and toxic effects of drugs; MDC 22: burns; MDC 23: factors influencing health status and other contacts with health services. 


\section{FIGURE 2}

Heat map showing the variation in standardised residual for total antibiotic consumption in 34 public acute hospitals, Ireland, 2006-2014

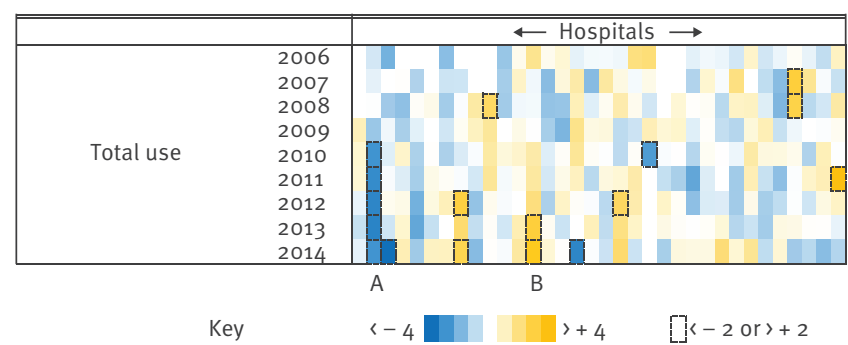

\section{Data sources}

Clinical antimicrobial dispensary data from hospital pharmacy systems were extracted and converted into defined daily doses (DDD) using the World Health Organization (WHO) Anatomical Therapeutic Chemical (ATC) classification method [9] via the MicroB secure online healthcare data analytical system [10]. Drugs dispensed to non-acute or non-inpatient areas were excluded. The rates for antibiotics were expressed as DDD per 100 bed-days used (BDU) and grouped into the following outcome variables:

1. Carbapenems, which included agents such as meropenem,

\section{Fluoroquinolones such as ciprofloxacin,}

3. Glycopeptides such as vancomycin and teicoplanin (excluding oral use),

4. Macrolides such as erythromycin,

5. Penicillins with enzyme inhibitors such as amoxicillin/clavulanic acid,

6. Third-generation cephalosporins such as cefotaxime, 7. Total antibiotic use, all systemic anti-bacterial agents.

Hospital In-patient Enquiry (HIPE) data following patients' discharge or death in the hospital were used to obtain aggregate annual patient administration variables from 2006 to 2014 for all participating hospitals. These were accessed through the Health Intelligence Ireland secure online healthcare data analytical system [11]. Data on non-inpatients (day cases and outpatients) were excluded from the analysis.

\section{Statistical Analysis}

R software was used for all statistical analyses [12]. We constructed log-normal regression models for each of the seven outcome variables using a stepwise forward selection method to identify risk and protective variables [13]. Collinear variables were removed following each selection. A categorical variable representing year was also entered.

Results for these models are reported using incidence rate ratios (coefficient estimate) and $95 \%$ confidence intervals for each outcome variable. Coefficients of variation of the root mean squared errors (CV-RMSE) are reported for each model.

Satisfactory models were used to generate expected values of antibiotic consumption for all facilities for each year in the study period, given the patient administration parameters for the facilities during the relevant time points. The difference between the observed antibiotic use and the estimated use is the residual, and the standardised residual is a ratio of the residual divided by the standard deviation of the residuals. Data points for any facility-year combination that had standardised residual values of less than -2 or greater than +2 were considered as having lower or higher than expected consumption, as estimated by the statistical model, respectively.

\section{Results}

Descriptive analysis

The total antibiotic usage rate for the 34 participating public acute hospitals decreased from 82.5 in 2006 to $80.0 \mathrm{DDD} / 100 \mathrm{BDU}$ in 2009 , and then increased to 89.2 DDD/100 BDU in 2014. Rates for carbapenems, glycopeptides and penicillins with enzyme inhibitors increased, those for macrolides and third-generation cephalosporins stayed level and those for fluoroquinolones decreased between 2006 and 2014 (Table 1).

A heat map of the 29 explanatory variables grouped into eight sections is shown in Figure $1 \mathrm{~A}$ and the 23 major diagnostic categories (MDC) in Figure 1B [14]. Each section for each hospital represents $100 \%$ of all discharged patients over the entire study period. Note that while the figures show combined values for all nine years for each hospital, individual data points for each year, hospital and explanatory variable were used in the regression analysis.

The proportion of patients aged 30 to 49 years ranged from $14 \%$ to $31 \%$ between hospitals and accounted for the highest proportions of all discharged patients, i.e. $22 \%$ of the all patients. The age group five to 14 years represented the lowest proportion of patients. The proportion of female patients ranged from $45 \%$ to $66 \%$ between hospitals and overall, $54 \%$ of all patients were female.

The most common type of admission was emergency, representing $70 \%$ of all discharges. Note that other admission types includes newborn and maternity admission. Among the admission sources, home was the most common type (88-97\%), while 'other source type' (prison, psychiatric unit or temporary residence) was the least common at $0-2 \%$. Among discharge 
TABLE 1

Consumption rates of five antibiotic groups and total antibiotics, Ireland, 2006-2014

\begin{tabular}{|c|c|c|c|c|c|c|c|}
\hline Year & Carbapenems & Fluoroquinolones & Glycopeptides & Macrolides & $\begin{array}{l}\text { Penicillins with } \\
\text { enzyme inhibitors }\end{array}$ & $\begin{array}{l}\text { Third-generation } \\
\text { cephalosporins }\end{array}$ & Total antibiotics \\
\hline 2006 & $1.2(0.1-4.9)$ & $10.3(5.0-30.0)$ & $2.4(0.2-7.0)$ & $12.3(5.4-20.3)$ & $20.5(14.5-36.5)$ & $1.9(0.3-3.7)$ & $82.5(56.6-118.1)$ \\
\hline 2007 & $1.2(0.0-3.0)$ & $10.2(6.2-27.8)$ & $2.3(0.4-4.7)$ & $11.7(6.3-20.5)$ & $21.4(13.3-38.7)$ & $1.6(0.6-3.2)$ & $80.6(61.6-105.8)$ \\
\hline 2008 & $1.9(0.1-6.2)$ & $8.7(5.3-28.1)$ & $2.6(0.3-6.5)$ & $11.8(6.5-20.3)$ & $22.1(11.6-40.9)$ & $1.6(0.3-3$ & $81.9(58$ \\
\hline 2009 & $2.3(0.1-6.7)$ & $6.5(0.6-26.2)$ & $2.9(0.2-7.2)$ & $11.0(5.8-20.2)$ & $22.6(14.6-39.4)$ & $1.5(0.4-3.0)$ & $80.0(63.3-112.8)$ \\
\hline 2010 & $2.6(0.4-7.9)$ & $6.1(1.6-11.7)$ & $3.0(0.4-7.8)$ & $11.4(5.6-21.8)$ & $24.0(14.3-38.9)$ & $1.6(0.4-3.7)$ & $83.4(63.0-124.9)$ \\
\hline 2011 & $2.6(0.2-7.5)$ & $6.2(2.5-12.1)$ & $3.2(0.6-8.0)$ & $12.4(5.9-23.2)$ & $26.2(18.2-42.5)$ & $1.6(0.7-3.4)$ & $87.9(67.0-135.6)$ \\
\hline 2012 & $3.0(0.5-9.4)$ & $6.3(2.7-12.5)$ & $3.0(0.4-5.2)$ & $12.7(5.9-27.9)$ & $27.4(19.7-42.9)$ & $1.7(0.2-4.0)$ & $88.6(66.6-126.7)$ \\
\hline 2013 & $3.7(0.2-9.6)$ & $5.9(2.5-11.0)$ & $3.2(0.5-5.4)$ & $12.2(4.8-25.5)$ & $27.0(19.7-40.6)$ & $1.6(0.1-3.8)$ & $87.3(62.3-114.8)$ \\
\hline 2014 & $4.1(0.5-9.0)$ & $5.9(2.6-11.5)$ & $3.5(0.6-5.7)$ & $12.2(3.3-21.4)$ & $26.9(16.1-40.9)$ & $1.8(0.2-4.3)$ & $89.2(45.7-129.1)$ \\
\hline
\end{tabular}

Rates are given in defined daily doses per 100 bed-days used, with minimum-to-maximum range in parentheses.

locations, home was again the most common type (73$92 \%$ ), while 'other location type' (prison, psychiatric unit or rehabilitation facility) was the least common at $0-2 \%$. Just over half of the patients ( $58 \%$ ) did not have private health insurance and the proportion of patients under a public payment scheme ranged from $36 \%$ to $79 \%$ per hospital.

Length of stay of one day (overnight) ranged from 14\% to $31 \%$, while a larger proportion stayed for between two to four days $(28-45 \%)$; the remainder stayed five days or longer $(28-55 \%)$. Overall, $7 \%$ of the patients had a stay in intensive care. Diseases and disorders of the circulatory system (MDC-5) and diseases and disorders of the digestive system (MDC-6) were the most common MDC overall at $15 \%$ each. Diseases and disorders of the respiratory system (MDC-4) were also frequent at $13 \%$. Of interest for antibiotic consumption are infectious and parasitic diseases (MDC-18) which was uncommon at just over $1 \%$.

\section{Regression analysis}

The final seven regression models for the antimicrobial groups are shown in Table 2. Note that only the variables that had a statistically significant association with any of the antimicrobial groups are shown. The model performance indicator, CV-RMSE, for total antibiotic use was only $11 \%$ indicating this to be a very good model. However, the CV-RMSE for fluoroquinolones was $36 \%$ and $31 \%$ for third-generation cephalosporins, indicating these to be poor models. The remaining models were adequate.

Different age groups were associated with increased risk of consumption of the different antibiotic groups. In particular, there was a high degree of association between decreased use of carbapenem and hospitals with a higher proportion of patients in the age group of five to 14 year-olds. Female sex was not significantly associated with any of the indicators of consumption, and neither was admission type (emergency, elective or other admission types).

Hospitals that had a higher proportion of patients admitted from 'other source type' had much reduced consumption of carbapenems, fluoroquinolones, glycopeptides, macrolides and total antibiotics. Similarly, hospitals that had a higher proportion of patients discharged to 'other location type' had much reduced consumption of glycopeptides, macrolides, third-generation cephalosporins and total antibiotics.

While only $0.1 \%$ of patients overall were classed under the MDC for burns (MDC 22), the category was associated with increased use of carbapenems and glycopeptides and with reduced use of fluoroquinolones and third-generation cephalosporins. The group of infectious and parasitic diseases (MDC 18) was associated with increased use of fluoroquinolones, penicillins with enzyme inhibitors, third-generation cephalosporins and total antibiotics.

Year as a categorical variable was significant for consumption of two antibiotic groups: carbapenems, which increased, and fluoroquinolones, which decreased over the study period. Two individual year values for macrolides (2009 and 2010) and one for total use (2009) were significant decreases.

\section{Outliers}

Figure 2 shows the variation in standardised residuals. Each data point with a standardised residual greater than +2 represented a time period of overuse of antibiotics at a particular hospital that was significantly greater than would be expected given the individual hospital's patient profile. Similarly, standardised residuals lower than -2 represented periods of significant underuse. For example, the hospital labelled A exhibited a reduction in consumption larger than expected for the patient profile of that hospital, and conversely, the hospital labelled B showed an overall increase. 


\section{TABLE 2A}

Incidence rate ratios of antibiotic consumption in public acute hospitals with $95 \%$ confidence intervals for the final seven regression models with percent CV-RMSE values, Ireland, 2006-2014

\begin{tabular}{|c|c|c|c|c|}
\hline Outcome variable & \multicolumn{2}{|c|}{ Significant variables } & Incidence rate ratio $(95 \% \mathrm{Cl})$ & CV-RMSE \\
\hline \multirow{22}{*}{ Carbapenem usage } & \multirow{4}{*}{ Age groups (years) } & $\leq 4$ & $1.09(1.06-1.12)$ & \multirow{22}{*}{$21 \%$} \\
\hline & & $5-14$ & $0.94(0.91-0.96)$ & \\
\hline & & $75-84$ & $0.96(0.95-0.97)$ & \\
\hline & & $\geq 85$ & $0.83(0.81-0.86)$ & \\
\hline & \multirow{2}{*}{ Admission source type } & Acute hospital & $0.54(0.45-0.64)$ & \\
\hline & & Other source type & $1.10(1.06-1.13)$ & \\
\hline & Discharge location type & Other location type & $1.01(1.01-1.02)$ & \\
\hline & Intensive care & $\operatorname{In~ICU}$ & $1.02(1.00-1.03)$ & \\
\hline & \multirow{7}{*}{$\begin{array}{l}\text { Major diagnostic } \\
\text { category }\end{array}$} & $M D C_{3}$ & $0.92(0.90-0.94)$ & \\
\hline & & $M D C_{5}$ & $0.98(0.96-1.00)$ & \\
\hline & & MDC 6 & $0.94(0.92-0.95)$ & \\
\hline & & MDC 9 & $1.09(1.05-1.14)$ & \\
\hline & & MDC 10 & $0.72(0.65-0.80)$ & \\
\hline & & MDC 11 & $1.11(1.08-1.15)$ & \\
\hline & & MDC 22 & $1.44(1.12-1.86)$ & \\
\hline & \multirow{7}{*}{ Year } & 2008 & $1.46(1.16-1.85)$ & \\
\hline & & 2009 & $1.77(1.41-2.23)$ & \\
\hline & & 2010 & $1.94(1.55-2.43)$ & \\
\hline & & 2011 & $1.95(1.56-2.45)$ & \\
\hline & & 2012 & $2.20(1.76-2.76)$ & \\
\hline & & 2013 & $2.35(1.87-2.95)$ & \\
\hline & & 2014 & $2.44(1.94-3.07)$ & \\
\hline \multirow{20}{*}{ Fluoroquinolone usage } & Age group (years) & $\geq 85$ & $0.97(0.94-1.00)$ & \multirow{20}{*}{$36 \%$} \\
\hline & Admission source type & Acute Hospital & $0.72(0.64-0.82)$ & \\
\hline & Length of stay (days) & 1 & $0.98(0.97-0.99)$ & \\
\hline & Intensive care & $\operatorname{In} \mathrm{ICU}$ & $1.02(1.01-1.04)$ & \\
\hline & \multirow{10}{*}{$\begin{array}{l}\text { Major diagnostic } \\
\text { category }\end{array}$} & $M D C_{5}$ & $1.06(1.04-1.08)$ & \\
\hline & & MDC 6 & $1.06(1.04-1.08)$ & \\
\hline & & $\mathrm{MDC}_{7}$ & $1.07(1.03-1.12)$ & \\
\hline & & MDC 8 & $1.03(1.02-1.04)$ & \\
\hline & & MDC 9 & $1.03(1.00-1.06)$ & \\
\hline & & MDC 14 & $1.03(1.02-1.04)$ & \\
\hline & & MDC 18 & $1.12(1.03-1.22)$ & \\
\hline & & MDC 19 & $1.09(1.05-1.12)$ & \\
\hline & & MDC 20 & $1.16(1.07-1.26)$ & \\
\hline & & MDC 22 & $0.68(0.48-0.97)$ & \\
\hline & \multirow{6}{*}{ Year } & 2009 & $0.72(0.62-0.85)$ & \\
\hline & & 2010 & $0.61(0.51-0.73)$ & \\
\hline & & 2011 & $0.67(0.56-0.80)$ & \\
\hline & & 2012 & $0.75(0.62-0.90)$ & \\
\hline & & 2013 & $0.75(0.62-0.91)$ & \\
\hline & & 2014 & $0.73(0.60-0.89)$ & \\
\hline
\end{tabular}

CI: confidence interval; CV-RMSE: coefficients of variation of the root mean squared errors; ICU: intensive care unit; MDC: major diagnostic category. Only the variables that had a statistically significant association with any of the antimicrobial groups are shown.

For the list of diseases in the different MDC, see Figure 1.

While this method of visualisation can be applied to subclasses of antibiotics, only the data from the model for total use is shown in Figure 2 as this model had the best model performance indicator, a CV-RMSE of $11 \%$.

\section{Discussion}

Our analysis identified three aspects of surveillance of hospital antimicrobial consumption: it identified factors that are important in driving antimicrobial use; it identified antibiotic groups for which the changes in consumption rate occur faster than could be explained by changes in patient profiles at the individual hospital alone; and it identified outliers so that stewardship strategies can be followed at those facilities to improve patient care. 
Incidence rate ratios of antibiotic consumption in public acute hospitals with $95 \%$ confidence intervals for the final seven regression models with percent CV-RMSE values, Ireland, 2006-2014

\begin{tabular}{|c|c|c|c|c|}
\hline Outcome variable & \multicolumn{2}{|c|}{ Significant variables } & \multirow{2}{*}{$\frac{\text { Incidence rate ratio }(95 \% \mathrm{Cl})}{1.02(1.01-1.03)}$} & \multirow{2}{*}{ CV-RMSE } \\
\hline \multirow{15}{*}{ Glycopeptide usage } & Age group (years) & $50-64$ & & \\
\hline & \multirow{3}{*}{ Admission source type } & Acute Hospital & $0.90(0.77-1.05)$ & \multirow{14}{*}{$23 \%$} \\
\hline & & Other healthcare facility & $1.16(1.09-1.24)$ & \\
\hline & & Other source type & $1.08(1.04-1.11)$ & \\
\hline & \multirow{2}{*}{ Discharge location type } & Acute hospital & $0.95(0.93-0.96)$ & \\
\hline & & Other healthcare facility & $0.88(0.82-0.95)$ & \\
\hline & Intensive care & In ICU & $1.03(1.01-1.04)$ & \\
\hline & \multirow{8}{*}{$\begin{array}{l}\text { Major diagnostic } \\
\text { category }\end{array}$} & $M D C 2$ & $1.04(1.01-1.08)$ & \\
\hline & & MDC 6 & $0.98(0.96-0.99)$ & \\
\hline & & MDC 10 & $0.93(0.87-0.99)$ & \\
\hline & & MDC 11 & $1.10(1.07-1.14)$ & \\
\hline & & MDC 12 & $0.92(0.85-1.00)$ & \\
\hline & & MDC 17 & $1.32(1.24-1.40)$ & \\
\hline & & MDC 21 & $0.89(0.83-0.95)$ & \\
\hline & & MDC 22 & $1.71(1.29-2.26)$ & \\
\hline \multirow{12}{*}{ Macrolide usage } & \multirow{2}{*}{ Age groups (years) } & $50-64$ & $1.02(1.02-1.03)$ & \multirow{12}{*}{$20 \%$} \\
\hline & & $75-84$ & $0.99(0.98-0.99)$ & \\
\hline & Admission source type & Acute hospital & $0.88(0.83-0.95)$ & \\
\hline & Discharge location type & Other healthcare facility & $0.94(0.89-1.00)$ & \\
\hline & \multirow{6}{*}{$\begin{array}{l}\text { Major diagnostic } \\
\text { category }\end{array}$} & MDC 2 & $0.93(0.90-0.96)$ & \\
\hline & & $M D C_{3}$ & $0.99(0.98-1.00)$ & \\
\hline & & MDC 4 & $1.03(1.02-1.04)$ & \\
\hline & & $M D C_{5}$ & $0.99(0.99-1.00)$ & \\
\hline & & $M D C_{9}$ & $0.96(0.94-0.98)$ & \\
\hline & & MDC 11 & $0.96(0.95-0.98)$ & \\
\hline & \multirow{2}{*}{ Year } & 2009 & $0.84(0.76-0.93)$ & \\
\hline & & 2010 & $0.90(0.81-0.99)$ & \\
\hline \multirow{8}{*}{ Usage of penicillins with enzyme inhibitor } & Admission source type & Other healthcare facility & $0.95(0.92-0.98)$ & \multirow{8}{*}{$19 \%$} \\
\hline & Discharge location type & Other location type & $0.99(0.99-1.00)$ & \\
\hline & Length of stay (days) & $2-4$ & $0.99(0.99-1.00)$ & \\
\hline & \multirow{5}{*}{$\begin{array}{l}\text { Major diagnostic } \\
\text { category }\end{array}$} & $M D C_{2}$ & $0.93(0.91-0.96)$ & \\
\hline & & $\mathrm{MDC}_{3}$ & $1.01(1.00-1.01)$ & \\
\hline & & MDC 4 & $1.02(1.01-1.03)$ & \\
\hline & & MDC 9 & $0.98(0.96-1.00)$ & \\
\hline & & MDC 18 & $1.05(1.00-1.10)$ & \\
\hline
\end{tabular}

$\mathrm{Cl}$ : confidence interval; CV-RMSE: coefficients of variation of the root mean squared errors; ICU: intensive care unit; MDC: major diagnostic category. Only the variables that had a statistically significant association with any of the antimicrobial groups are shown.

For the list of diseases in the different MDC, see Figure 1.

On the first aspect, the main patient profile factors including MDC, and their variation both over time and across different facilities, may explain the dynamics that are evident in hospital antimicrobial consumption in Ireland. The range of factors that were significant for the different antimicrobial groups shows that using only a single factor such as the cost-based case mix index would not have been adequate [15]. Additional factors that could have been employed include clinical services parameters in conjunction with administration data or variables that define patient profiles at a finer level such as specific diagnosis/procedure codes $[5,6]$.
On the second aspect, fluoroquinolones were the only antimicrobials in this study for which consumption decreased. There has been a concerted effort since 2008 by pharmacists in Ireland to reduce fluoroquinolone use as a whole and to switch to oral preparations as fluoroquinolones have a good bioavailability [16]. The increase in carbapenems is a concern as carbapenem-resistant Enterobacteriaceae are becoming more frequent across Europe [17]. All hospitals and the health service in Ireland have a collective responsibility to ensure that these increases are curtailed. 
Incidence rate ratios of antibiotic consumption in public acute hospitals with $95 \%$ confidence intervals for the final seven regression models with percent CV-RMSE values, Ireland, 2006-2014

\begin{tabular}{|c|c|c|c|c|}
\hline Outcome variable & \multicolumn{2}{|c|}{ Significant variables } & Incidence rate ratio $(95 \% \mathrm{Cl})$ & CV-RMSE \\
\hline \multirow{13}{*}{ Third-generation cephalosporin usage } & \multirow{2}{*}{ Age group (years) } & $\leq 4$ & $1.03(1.00-1.06)$ & \multirow{13}{*}{$31 \%$} \\
\hline & & $5-14$ & $1.03(1.01-1.05)$ & \\
\hline & Admission source type & Other source type & $1.10(1.06-1.14)$ & \\
\hline & \multirow{2}{*}{ Discharge location type } & Other healthcare facility & $0.80(0.73-0.88)$ & \\
\hline & & Other location type & $1.00(1.00-1.01)$ & \\
\hline & Length of stay (days) & 1 & $0.97(0.96-0.98)$ & \\
\hline & \multirow{7}{*}{$\begin{array}{l}\text { Major diagnostic } \\
\text { category }\end{array}$} & $M D C_{1}$ & $1.06(1.04-1.08)$ & \\
\hline & & $\mathrm{MDC}_{3}$ & $0.97(0.95-0.98)$ & \\
\hline & & $M D C_{7}$ & $1.06(1.03-1.10)$ & \\
\hline & & MDC 10 & $0.87(0.79-0.95)$ & \\
\hline & & MDC 13 & $1.11(1.08-1.14)$ & \\
\hline & & MDC 18 & $1.28(1.18-1.40)$ & \\
\hline & & MDC 22 & $0.42(0.29-0.62)$ & \\
\hline \multirow{14}{*}{ Total antibiotic usage } & \multirow{2}{*}{ Admission source type } & Acute hospital & $0.91(0.87-0.95)$ & \multirow{14}{*}{$11 \%$} \\
\hline & & Other source type & $1.03(1.02-1.04)$ & \\
\hline & Discharge location type & Other healthcare facility & $0.94(0.91-0.97)$ & \\
\hline & Intensive care & $\ln \mathrm{ICU}$ & $1.01(1.01-1.01)$ & \\
\hline & \multirow{9}{*}{$\begin{array}{l}\text { Major diagnostic } \\
\text { category }\end{array}$} & $M \mathrm{MC}_{1}$ & $0.99(0.98-0.99)$ & \\
\hline & & $M D C_{2}$ & $0.98(0.97-1.00)$ & \\
\hline & & $M D C_{3}$ & $0.99(0.99-0.99)$ & \\
\hline & & $\mathrm{MDC}_{7}$ & $1.02(1.01-1.03)$ & \\
\hline & & MDC 8 & $1.00(1.00-1.01)$ & \\
\hline & & MDC 10 & $0.97(0.95-1.00)$ & \\
\hline & & MDC 17 & $1.02(1.00-1.04)$ & \\
\hline & & $M D C 18$ & $1.04(1.01-1.08)$ & \\
\hline & & MDC 20 & $1.08\left(1.05^{-1.12)}\right.$ & \\
\hline & Year & 2009 & $0.93(0.87-0.99)$ & \\
\hline
\end{tabular}

$\mathrm{Cl}$ : confidence interval; CV-RMSE: coefficients of variation of the root mean squared errors; ICU: intensive care unit; MDC: major diagnostic category. Only the variables that had a statistically significant association with any of the antimicrobial groups are shown. For the list of diseases in the different MDC, see Figure 1.

On the last aspect of outliers, our analysis showed that there were hospitals that had consistently higher antibiotic consumption than would be expected given the characteristics of patients cared for in those hospitals. It is likely that these few hospitals have services that were not included in the parameters of our models. It is important to address the presence of and adherence to antibiotic prescribing policies in these institutions.

Our analysis has limitations. Firstly, there is a debate about how to appropriately measure antibiotics usage. We selected the WHO ATC/DDD system as it is the one chosen by the European Surveillance of Antimicrobial Consumption Network (ESAC-Net). Furthermore, direct measures of antibiotic usage such as days of therapy could not be used as the pharmacy computer systems used in Ireland do not yet support it, unlike hospitals elsewhere [18]. The second limitation is the choice of denominator to express rates of use, of which there are also different viewpoints in the literature such as using number of admissions or discharges, or bed-days (or patient-days) used [19]. We selected bed-days used, as this denominator takes into account the average length of stay. However, given the strong association between length of stay of one day and total antibiotic use, number of admissions may be a more appropriate denominator. The third limitation is the possible presence of coding errors, and although hospital administration data in Ireland are increasingly used for research purposes, further validation is warranted [7]. The fourth limitation is the choice of regression method. Again, a variety of approaches have been attempted in the literature, ranging from indirect/direct standardisation, Poisson and negative-binomial regression, to simple linear regression $[15,18,20]$. We selected log-normal regression as the data fitted this distribution and satisfied its assumptions. Generalised estimating equations or mixed effects models were not required as it was the aim of our study to show differences between hospitals and adjust them via the explanatory variables [21]. The choice of modelling method also allowed for the use of conventional methods of analysis rather 
than employing complex procedures to compensate for overdispersion. However, the CV-RMSE for two of the models were very large and use of additional explanatory parameters is warranted. The last limitation is the sample size of only 34 hospitals. Even after including private and single-speciality hospitals, the population base would remain the overriding limit for any study conducted in Ireland. Extending the methodology to include other countries would be the only way to overcome this limitation.

Based on the findings of this study we recommend that the national guidance documents for antimicrobial stewardship should be updated to strengthen prescribing practice for carbapenems in particular and to incorporate a mechanism to ensure good adhere to antibiotic prescribing. We also recommend that performance-linked measures are put in place to ensure that when hospitals demonstrate reduction in the use of one antibiotic group, this does not lead to increases in another group of antibiotics. However, high antibiotic use among outliers may not imply poor performance and the hospitals not found to be outliers may still have substantial inappropriate use. Therefore, the findings of this study should be used in conjunction with other information and as part of a broader stewardship strategy. Finally, we suggest that a Europe-wide hospital antimicrobial study based on a unified methodology of risk adjustment is undertaken that takes into account the limitations of this and other similar studies. Risk adjustment may even be required to compare the wide variation in hospital antibiotic consumption as driven by diverse healthcare delivered to the populations in different jurisdictions.

In conclusion, patient-based administrative data variables are useful for adjusting hospital antibiotic consumption rates, although additional variables relating to clinical services should also be employed.

\section{Acknowledgements}

We thank all of the hospitals that provided the antimicrobial consumption data and the Irish Antimicrobial Pharmacists Group for their advice. We thank HSE Business Information Unit for providing the denominator data. We acknowledge the Economic and Social Research Institute and HSE Healthcare Pricing Office for providing Hospital In-Patient Enquiry data.

\section{Conflict of interest}

None declared.

\section{Authors' contributions}

AO was involved in acquisition and analysis of the data and wrote the manuscript. FD and $\mathrm{HJ}$ were involved in the analysis of HIPE data, and reviewed the manuscript. RC initiated the study, was involved in study design and reviewed the manuscript. All authors read and approved the final manuscript.
References

1. Dumartin C, L'Hériteau F, Péfau M, Bertrand X, Jarno P, Boussat $S$, et al. Antibiotic use in 530 French hospitals: results from a surveillance network at hospital and ward levels in 2007. I Antimicrob Chemother. 2010;65(9):2028-36. DOI: 10.1093/jac/ dkq228 PMID: 20581121

2. Blix HS, Hartug S. Hospital usage of antibacterial agents in relation to size and type of hospital and geographical situation.Pharmacoepidemiol Drug Saf. 2005;14(9):647-9. DOI: 10.1002/pds.1080 PMID: 15700320

3. Consumption of antibiotics in public acute hospitals in Ireland data for first half of 2013. Dublin: Health Protection Surveillance Centre; [Accessed: 22 Aug 2015]. Available from: http://www.hpsc.ie/A-Z/MicrobiologyAntimicrobialResistance/ EuropeanSurveillanceofAntimicrobialConsumptionESAC/ SurveillanceReports/HospitalAntibioticUse/File,14350,en.pdf

4. Iezzoni LI. Reasons for risk adjustment. In LI lezzoni. Risk adjustment for measuring health care outcomes. 3rd ed. Chicago: Health Administration Press; 2003. Available from: http://intqhc.oxfordjournals.org/content/16/2/181

5. Polk RE, Hohmann SF, Medvedev S, Ibrahim O. Benchmarking risk-adjusted adult antibacterial drug use in 70 US academic medical center hospitals.Clin Infect Dis. 2011;53(11):1100-10. DOI: 10.1093/cid/cir672 PMID: 21998281

6. Rajmokan M, Morton A, Marquess J, Playford EG, Jones M. Development of a risk-adjustment model for antimicrobial utilization data in 21 public hospitals in Queensland, Australia (2006-11).J Antimicrob Chemother. 2013;68(10):2400-5.PMID: 23689029

7. Wiley MM. Using HIPE data as a research and planning tools: limitations and opportunities: A Response.Ir J Med Sci. 2005;174(2):52-7. DOI: 10.1007/BF03169130

8. European Centre for Disease Prevention and Control (ECDC). Surveillance of antimicrobial consumption in Europe 2012. Stockholm: ECDC; 2014. Available from: http://ecdc.europa. eu/en/publications/Publications/antimicrobial-consumptioneurope-esac-net-2012.pdf

9. ATC/DDD Index 2016. Oslo: WHO Collaborating Centre for Drug Statistics Methodology; 2015. Available from: http://www. whocc.no/atc_ddd_index

10. Donlon S, Houlden M, Oza A. HPSC launches new web-based system to manage hand-hygiene compliance and antibiotic usage data. Epi Insight. 2013; 14(2):1. Available from: http:// ndsc.newsweaver.ie/epiinsight/1hoijtrdr8f?a $=1 \& p=31757935$ $\& \mathrm{t}=17517774$

11. Health Atlas Ireland. Dublin: Health Intelligence Ireland. [Accessed: Mar 2016]. Available from: https://www. healthatlasireland.ie/

12. R Core Team. R: A language and environment for statistical computing. Vienna: R Foundation for Statistical Computing; 2015. Available from: http://www.R-project.org/

13. Limpert E, Stahel WE, Abbt M. Log-normal distributions across the sciences: keys and clues.Bioscience. 2001;51(5):341-52. DOI: 10.1641/0006-3568(2001)051[0341:LNDATS]2.0.CO;2

14. Australian Institute of Health and Welfare. Australian refined diagnosis-related groups (AR-DRG) data cubes. Canberra: Australian Institute of Health and Welfare. [Accessed; 22 Aug 2015]. Available from: http://www.aihw.gov.au/hospitals-data/ ar-drg-data-cubes/

15. Kuster SP, Ruef C, Blliner AK, Ledergerber B, Hintermann A, Deplazes $C$, et al. Correlation between case mix and antibiotic use in hospitals. J Antimicrob Chemother. 2008;62:(4):837-42.

16. SARI Hospital Antimicrobial Stewardship Working Group. Guidelines for antimicrobial stewardship in hospitals in Ireland. Dublin; Health Protection Surveillance Centre; 2009. ISBN: 978-0-9551236-7-2.Available from: https:// www.hpsc.ie/A-Z/MicrobiologyAntimicrobialResistance/ InfectionControlandHAI/Guidelines/File,4116,en.pdf

17. Oza A, Burns K, Cunney R. Carbapenem use in hospitals has doubled in the last five years. Epi Insight. 2014;15(11):1. Available from: http://ndsc.newsweaver.ie/epiinsight/ig9up54i $182 ? a=1 \& p=48078701 \& t=17517774$

18. Kanerva M, Ollgren J, Lyytikäinen O, Agthe N, Mottonen T, Kauppinen $\mathrm{M}$, et al. Benchmarking antibiotic use in Finnish acute care hospitals using patient case-mix adjustment. I Antimicrob Chemother. 2011;66(11):2651-4. DOI: 10.1093/jac/ dkr333 PMID: 21846673

19. Amadeo B, Dumartin C, Robinson P, Venier AG, Parneix P, Gachie JP, et al. Easily available adjustment criteria for the comparison of antibiotic consumption in a hospital setting: experience in France. Clin Microbiol Infect. 2010;16(6):735-41. DOI: 10.1111/j.1469-0691.2009.02920.x PMID: 19778299

20. MacDougall C, Polk RE. Variability in rates of use of antibacterials among 130 US hospitals and risk-adjustment models for interhospital comparison. Infect Control Hosp 
Epidemiol. 2008;29(3):203-11. DOI: 10.1086/528810 PMID: 18257689

21. Hubbard AE, Ahern J, Fleischer NL, Van der Laan M, Lippman $\mathrm{SA}$, Jewell N, et al. To GEE or not to GEE: comparing population average and mixed models for estimating the associations

between neighborhood risk factors and health. Epidemiology. 2010;21(4):467-74. DOI: 10.1097/EDE.obo13e3181caeb9o PMID: 20220526

\section{License and copyright}

This is an open-access article distributed under the terms of the Creative Commons Attribution (CC BY 4.0) Licence. You may share and adapt the material, but must give appropriate credit to the source, provide a link to the licence, and indicate if changes were made.

This article is copyright of the authors, 2016. 